\title{
Whole-exome sequencing insights into pulmonary artery sarcoma mimicking pulmonary embolism: a case report and review
}

This article was published in the following Dove Press journal: OncoTargets and Therapy

\author{
Ying $\mathrm{Wu}^{\prime}$ \\ Jing Huang' \\ Qin Wang ${ }^{2}$ \\ Mei Zhang' \\ Yimin Luo' \\ Xihua Wang' \\ Xiaoli Zhu' \\ Hongbing Liu $^{2}$ \\ 'Department of Respiratory Medicine, \\ Zhongda Hospital, School of Medicine, \\ Southeast University, Nanjing 210009, \\ People's Republic of China; ${ }^{2}$ Department \\ of Respiratory Medicine, Jinling Hospital, \\ Nanjing University School of Medicine, \\ Nanjing 210002, People's Republic of \\ China
}

\begin{abstract}
Pulmonary artery sarcoma (PAS) is a rare and aggressive mesenchymal tumor that often mimics thromboembolic disease. Due to its rare and fatal nature, patients are often underdiagnosed or misdiagnosed. There is still no consensus regarding the diagnosis and treatment of PAS. We present a case of a 63 year old male misdiagnosed with pulmonary thromboembolism who received anticoagulant therapy. ${ }^{18} \mathrm{FDG}$ positron emission tomography (PET) integrated with computed tomography (PET/CT) and subsequent surgery led to the final diagnosis of PAS. Whole exome sequencing of the tissue identified the genetic alterations profile of PAS: copy number variation (CNV) of KIT and mutations of TP53, PIK3CA, $I L 7 R$ and $A T R$. Treated with chemotherapy followed by anlotinib, the patient's survival time was 8 months after firm diagnosis. To our knowledge, anlotinib used as a treatment for PAS has not been reported.
\end{abstract}

Keywords: pulmonary artery sarcoma, whole exome sequencing, anlotinib

\section{Introduction}

Pulmonary artery sarcoma (PAS) is a rare and lethal disorder of unknown etiology. ${ }^{1}$ Since being reported by Mandelstamm in 1923, fewer than 400 individual cases have been reported, and most of these cases were misdiagnosed before surgery or autopsy. Due to the rarity and fatality of PAS, there are no widely accepted diagnostic protocols and effective treatments available.

Herein, we present a case of PAS. Whole-exome sequencing (WES) was conducted to illustrate the phenotypic variation of PAS and may have implications for treatment. A literature review of published cases was also conducted.

\section{Case presentation}

A 63 year old male was presented with a 1-month history of cough. The patient had a history of allergic rhinitis 20 years ago. He had never smoked previously. A computed tomography (CT) scan of the chest revealed a faint infiltrative shadow in the right lower lung field (Figure 1A). The CRP level was $12.7 \mathrm{mg} / \mathrm{L}$ (normal range: 0-3.0). Other laboratory tests did not show any significant abnormalities, including those of $\operatorname{IgE}, \mathrm{D}$-dimer and tumor markers. The patient was diagnosed with pneumonia, and an anti-inflammatory was given. However, there was no clinical improvement after nearly one month. Repeated CT scans showed inflammation of the right pulmonary was progressing nearly one month later. The right
Department of Respiratory Medicine,

Southeast University, 87 Dingjiaqiao, Nanjing

210009 , People's Republic of China

Tel +862583262803

Email wangxihua@I26.com

Hongbing Liu

Department of Respiratory Medicine, Jinling

Hospital, Nanjing University School of

Medicine, 305 East Zhongshan Road,

Nanjing 210002, People's Republic of China

Tel +862580860592

Email netlhb@I26.com 


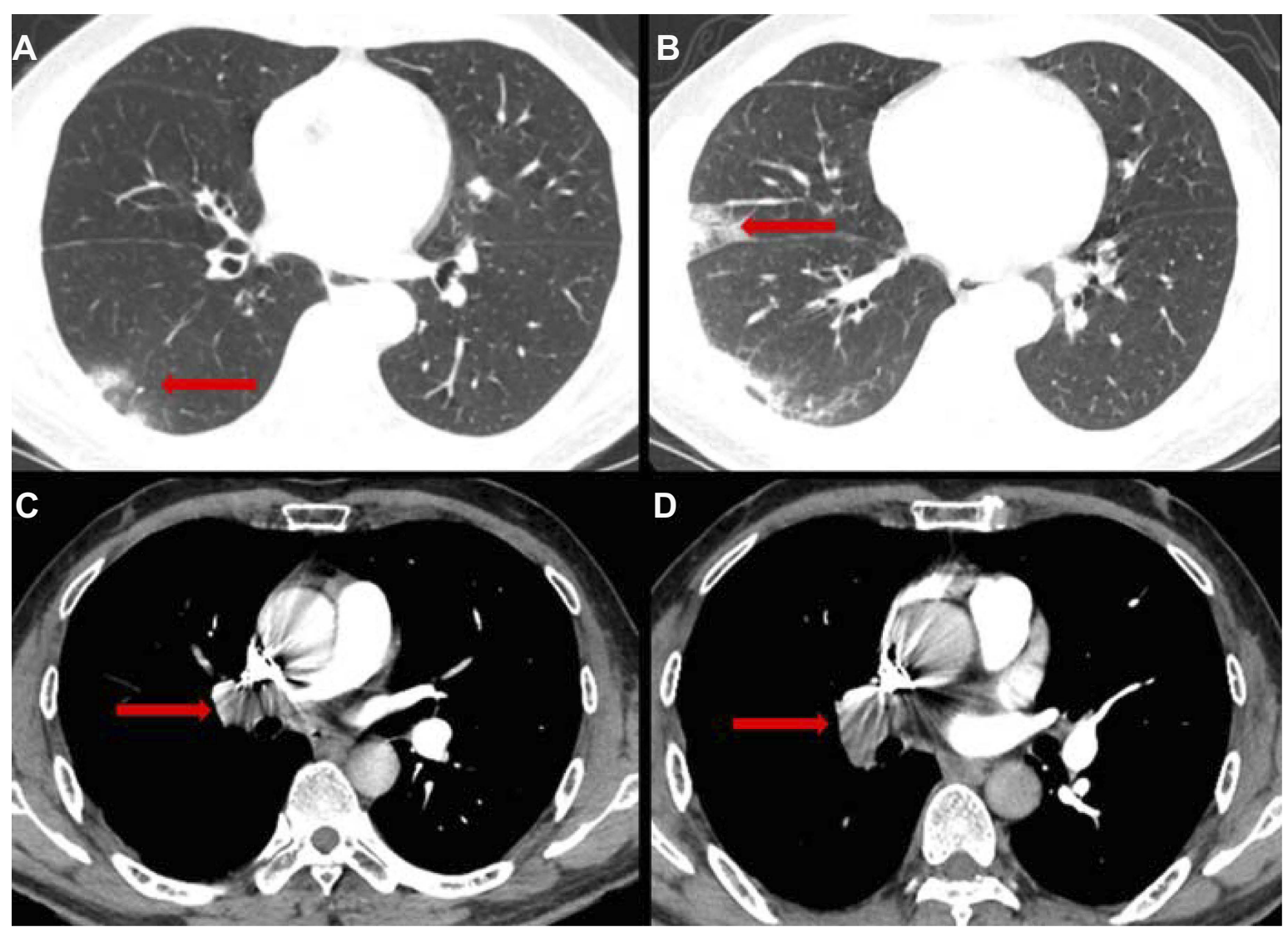

Figure I (A) CT scan of the chest demonstrated a faint infiltrative shadow in the right lower lung field (red arrow). (B) Repeated CT scan showed inflammation of the right pulmonary was progressing nearly one month later. The right lung is scattered in the flaky blurred shadow with an unclear boundary, which is obvious in the middle and lower lobes (red arrow). (C and D) CT pulmonary angiography revealed extensive intraluminal filling defects in the trunk and branches of the right pulmonary artery (red arrow). No filling defects were observed in the left pulmonary artery.

lung showed as scattered in the flaky blurred shadow with an unclear boundary, which is obvious in the middle and lower leaves. (Figure 1B)

Although the D-dimer and blood gas analyses were normal, CT pulmonary angiography was performed (Figure 1C and D). The initial CT pulmonary artery angiography revealed extensive intraluminal filling defects in the trunk and branches of the right pulmonary artery. No filling defects were observed in the left pulmonary artery. He was thus diagnosed with pulmonary thromboembolism (PTE). Then, he was given an anticoagulant treatment. Two months later, the patient's symptom aggravated and presented with chest pain, dyspnea on exertion and weight loss. The D-dimer value was still normal.

For further evaluation of a possible malignancy, ${ }^{18} \mathrm{FDG}$ positron emission tomography (PET) integrated with computed tomography (PET/CT) was performed. ${ }^{2}$ In the trunk and segmental of the right pulmonary artery, a strip-shaped intraluminal lesion with obvious increased FDG uptake was observed (SUVmax $=9.69$ ). Multiple plaques can be seen in the right lung with slightly increased FDG metabolism (SUVmax =1.64). A right pleural nodule with increased FDG uptake (SUVmax $=2.45$ ) was next to the 7 th rib on the right (Figure $2 \mathrm{~A}$ and $\mathrm{B}$ ). It strongly suggested a primary malignant tumor of the pulmonary artery. These findings implied a possible diagnosis of primary sarcoma of the pulmonary artery with metastases to the lungs and chest wall.

Video-assisted thoracoscopic surgery of the right pleural nodule was performed for the diagnosis. The metastatic lesion was located in the basal segment of the right lower lobe. There were swollen lymph nodes in the lungs, in the middle of the bronchi and upper longitudinal and subcarinal lymph nodes. Biopsy of the tumor from the basal segment of the right lower lobe was taken to determine the tumor etiology. The tumor mostly consisted of a population of malignant spindle cells. The patient tissue was fixed in $10 \%$ neutral buffered formalin solution for $24 \mathrm{hrs}$, followed by paraffin embedding. Standard immunostaining techniques were used to prepare the sections for histology and immunohistochemistry staining. Vimentin, Desmin, MDM2 and P53 


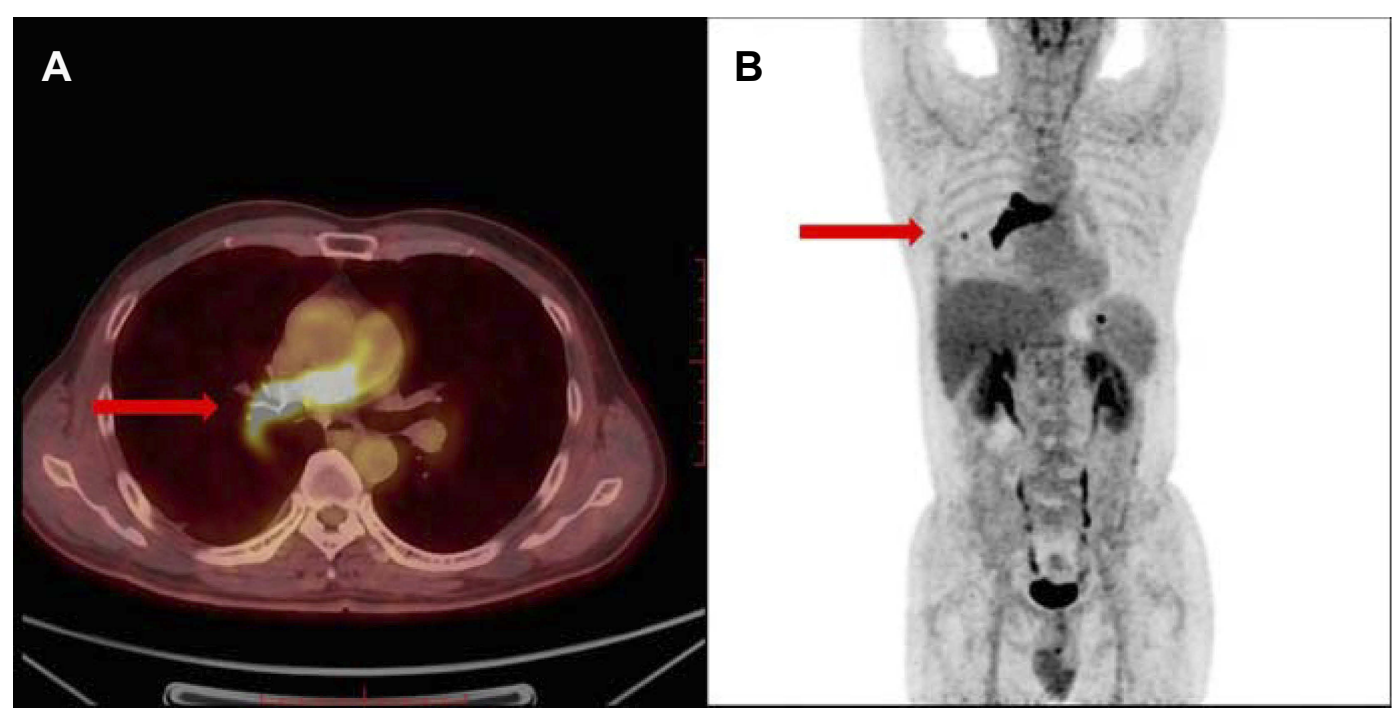

Figure 2 (A) Increased FDG uptake of PET/CT observed in an intraluminal lesion of the right pulmonary artery (red arrow in A, SUVmax 9.69). (B) Increased FDG uptake observed along the wall of the right lobar (red arrow in B, SUVmax 2.45).

were stained by using the following primary antibodies: antiVimentin (Dako., Glostrup, Denmark), anti-Desmin (Dako., Glostrup, Denmark), anti-MDM2 (Maixin, Fuzhou, China), and anti-P53 (Dako., Glostrup, Denmark). The secondary antibody (Dako., Glostrup, Denmark) was used. Tumor cells expressed Vimentin and Desmin by immunohistochemistry. These tumors also overexpress MDM2 and mutant P53 (Figure 3). In addition, the tumor cells showed negative immunostaining for TTF-1, Pan-CK, CD20, CD31, CD34, ERG, S-100, HMB45, SMA, Mum-1, MyoD1, and Myogenin. Typically, poorly differentiated tumors, such as PAS, are positive for vimentin. ${ }^{3}$ Thus, the tumor strongly positive for desmin and vimentin was suggestive of a diagnosis of subtype of PAS, which is recognized as "undifferentiated sarcoma".

We also conducted whole exome sequencing of the tissue to explore possible molecular abnormalities (Kunyuan Gene Technology Co., Ltd., Shanghai, China; www.singlera.com. cn). DNA in the patient's blood was considered baseline to reduce the influence of leukocytes. DNA was extracted from tissue and blood samples using a DNeasy Blood and Tissue Kit (Qiagen, Hilden, Germany) under the manufacturer's instructions and was subjected to WES. DNA was quantified using the Qubit 3.0 Fluorometer (Thermo Scientific, MA, USA). DNA was mechanically fragmented on an E220 focused ultrasonicator Covaris (Covaris, Woburn, MA, USA). DNA library preparation and exome capturing were performed using the xGen Exome Research Panel (Integrated DNA Technologies). After quality control and quantification by Agilent 2100 Bioanalyzer (Agilent Technologies) and
Qubit 3.0 Fluorometer (Thermo scientific, MA, USA), sequencing was undertaken using Illumina NovaSeq (llumina Inc, San Diego, CA, USA).

Overall, these genes are divided into five classes: targeted drug-related genes, genes related to chemotherapy, gene changes predicted by immunotherapy, genetic susceptibility genes and other genes. In targeted drug-related genes, KIT copy number variation (CNV) and TP53 and PIK3CA mutations were found. KIT CNV indicated KIT gene amplification (copy number=6). The $P I K 3 C A$ gene had missense mutations at the encoding sequence NM_006218.4:c.1345C $>$ A (p.P449T.) in exon 8 with an abundance of 43.9. The TP53 gene had mutations in the encoding sequence NM_000546.5:c.329G $>$ C (p.R110P) in exon 4 with an abundance of 62.1. Evaluation of the efficacy of immunological checkpoint inhibitors, such as tumor mutation burden (TMB), mismatch repair (MMR), and programmed death-ligand 1 (PD-L1), was all negative. This finding indicated that the treatment of immunological checkpoint inhibitors was unsuitable for the patient. The following drug metabolism-related polymorphisms were detected: NAD $(\mathrm{P}) \mathrm{H}$ quinone dehydrogenase 1 (NQO1) (rs1800566 GA), UDP glucuronosyltransferase family 1 member A1 (UGT1A1) (rs4148323 GG), methylenetetrahydrofolate reductase (MTHFR) (rs1801133 GG), and cytochrome P450 family 19 subfamily a member 1 (CYP19A1) (rs4646 AA). Unfortunately, there was no genetic variation in genetic susceptibility genes. Two other mutations were identified in this patient, namely, NM_001184.4:c.1732G >A (p.V578I) in the rad3-related 

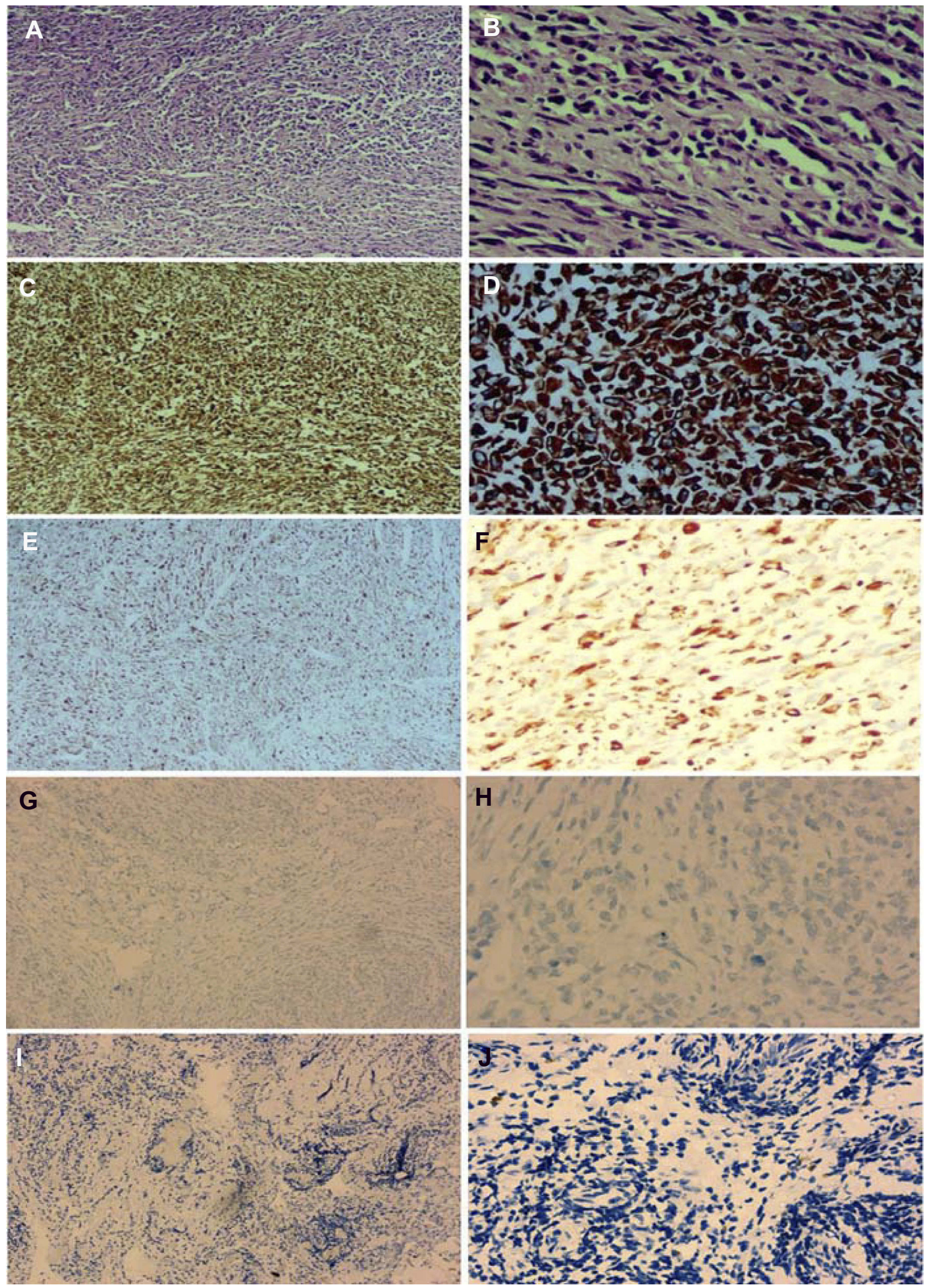

Figure 3 Hematoxylin-eosin staining and immunohistochemistry staining in tumor cells.

Notes: (A and B) Hematoxylin-eosin staining ( $\times 100$, and 400, respectively); (C and D) The tumor cells were positive for vimentin ( $\times 100$, and 400, respectively); (E and F) The tumor cells were positive for desmin ( $\times 100$, and 400, respectively); ( $\mathbf{G}$ and $\mathbf{H})$ The tumor cells were positive for MDM2 ( $\times 100$, and 400, respectively); (I and J) The tumor cells were positive for P53 $(\times 100$, and 400, respectively). 
protein (ATR) gene and NM_002185.5:c.731C $>$ T (p T244I) in the interleukin 7 receptor (IL7R) gene. (Table 1)

The patient did not want surgery treatment because of the risks associated with surgery. Thus, whole exome sequencing was performed to identify therapeutic target and genetic susceptibility genes. It seems that there was no specific target therapy or immunotherapy agent for him. Although the MTHFR rs 1801133 polymorphism indicated that the patient might be sensitive to pemetrexed, the patient received a chemotherapy regimen of pemetrexed. After two cycles of pemetrexed, clinical manifestations were exacerbated, and pulmonary artery pressure was as high as $114 \mathrm{mmHg}$ by echocardiography. In addition, a CT scan showed that right lung metastasis lesions of the patient became larger with newly liver metastasis and thoracic vertebra metastasis. (Figure 4A and B) Given the efficacy of anlotinib in sarcoma, the patient received anlotinib with a cycle of at a dose of $12 \mathrm{mg} /$ day for two weeks and then stopped for one week. More than one month later, right lung metastasis lesions were slightly smaller than before, and pulmonary artery pressure of $70 \mathrm{mmHg}$ was lower (Figure 4C and D). After four cycles of anlotinib, the patient refused anlotinib therapy because of severe nausea and liver and renal insufficiency. He also declined further evaluation. The patient died 8 months after diagnosis.

Written informed consent was obtained from the patient for this publication and the accompanying images and tables.

\section{Discussion}

The incidence of primary PAS is extremely low, accounting for $2 \%$ of all sarcomas. PAS is a rare and aggressive malignant tumor with intraluminal growth, subsequent vessel block, and proximal or remote metastases. Intimal sarcoma of the pulmonary artery has no specific symptoms at an early stage and has been easily misdiagnosed as pneumonia or PTE due to the difficulty of diagnosis from current imaging technology. ${ }^{4}$

Clinical manifestation has no significant specificity, including cough, pleuritic pain, dyspnea, hemoptysis, syncope, etc. When combined with pulmonary hypertension and right ventricular dysfunction, there may be cyanosis, jugular vein engorgement, hepatomegaly, edema of both lower extremities, etc. Our patient exhibited symptoms such as cough and pleuritic pain, which persisted for more than two months. A filling defect in the right pulmonary artery trunk was observed on a contrast-enhanced chest CT. The clinical and radiological findings of pulmonary artery angiosarcoma were similar to PTE. PTE is more acute, and include sudden shortness of breath, chest pain, hemoptysis and even syncope. D-dimer is significantly elevated, and serum tumor markers are normal in PTE. Anticoagulation or thrombolytic therapy is effective in PTE. However, in our patient, after anticoagulant therapy, pleuritic pain was not relieved, and symptoms of right ventricular dysfunction slowly appeared. In addition, Ddimer examinations were in the normal range a multiple number of times. Then, the alternative diagnosis of pulmonary artery sarcoma should be considered. When suspected pulmonary sarcoma is present, a more sensitive inspection method is needed, such as PET/CT. This patient underwent $\mathrm{PET} / \mathrm{CT}$ examination, suggesting a malignant occupying of the right pulmonary trunk and was finally diagnosed with pulmonary sarcoma after lobectomy.

Regularly, PET/CT has been shown to be useful for distinguishing between benign and malignant lesions and detecting distant metastasis. The utilization of ${ }^{18} \mathrm{FDG}$ in the diagnosis of PAS is still under investigation. Some case

Table I. The profile of gene mutation or copy number variation by a whole exome sequencing in the patient with pulmonary artery sarcoma

\begin{tabular}{|l|l|l|l|l|l|}
\hline \multirow{2}{*}{ Genes } & \multicolumn{2}{|l|}{ Mutations or CNV } & \multirow{2}{*}{ Abundance (\%) } \\
\cline { 2 - 6 } & Transcript ID. & Exon & Sequence & Coding \\
\hline ATR & NM_00II84.4 & 7 & c. I732G>A & P.V578I & 31.9 \\
IL7R & NM_002185.5 & 6 & c.73IC>T & P.T244I & 60.4 \\
PIK3CA & NM_006218.4 & 8 & c.1345C>A & P.P449T & 43.9 \\
TP53 & NM_000546.5 & 4 & c.329G>C & P.RIIOP & 62.1 \\
\hline KIT & CNV & & & 6 \\
\hline
\end{tabular}

Abbreviations: CNV, copy number variation; ATR, ataxia telangiectasia and rad3-related protein; IL7R, interleukin 7 receptor; PIK3CA, phosphatidylinositol-4,5-bisphosphate 3-kinase catalytic subunit alpha; TP53, tumor protein P53; KIT, KIT Proto-Oncogene Receptor Tyrosine Kinase. 


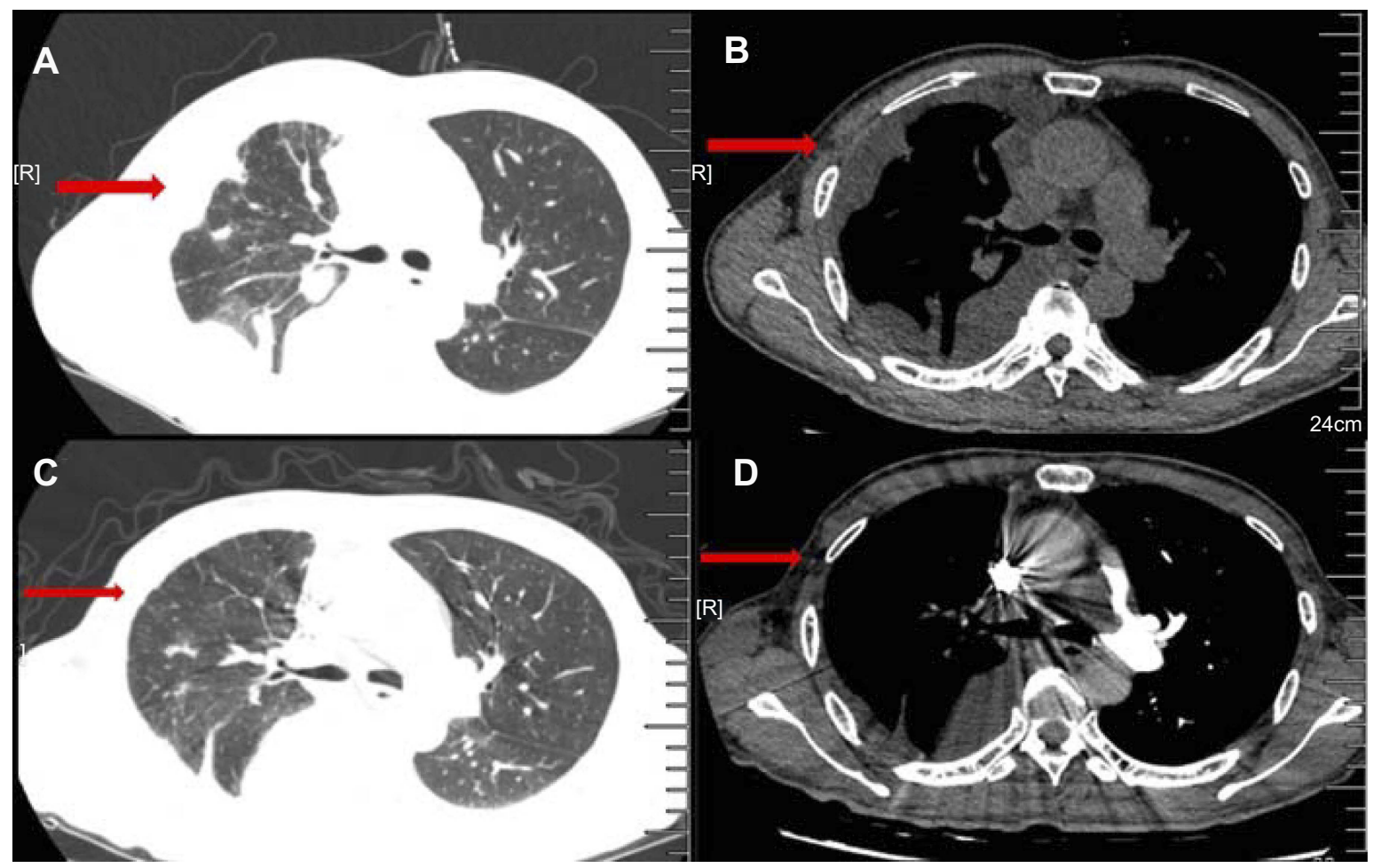

Figure 4 (A and B) After a chemotherapy regimen of pemetrexed, CT scan showed metastasis lesions in the right upper lobe and right lower lobe of the patient. (C and D) CT scan showed metastasis lesions in the right upper lobe and right lower lobe of the patient after two cycles of anlotinib. The red arrows represent the metastasis lesions in the right lung.

reports of PET/CT showed an intense hypermetabolism in the lesion and could differentiate whether the lesion was a thrombus or a malignancy, ${ }^{5-8}$ whereas other cases were also reported to show poor FDG uptake in PAS. ${ }^{9}$ A recent study of a large sample revealed that PET/CT could differentiate malignancy of the pulmonary artery from PTE and that increased ${ }^{18} \mathrm{FDG}$ uptake value is an accurate index for determining PA malignancy. ${ }^{10}$ As expected, the PET/CT results of the present case showed increased ${ }^{18} \mathrm{FDG}$ uptake in the intraluminal lesion of the right pulmonary artery (SUVmax 9.69) and right hilar nodule (SUVmax 2.45). Certainly, PET/ $\mathrm{CT}$ is easy to accurately detect the prevalence of metastases at intra- and extrapulmonary sites. However, well-designed large-scale studies are essential to confirm and validate the role of PET/CT in PAS in the near future.

Although it is difficult to obtain diagnostic material, a pathological examination is required to establish a definite diagnosis. So far, the majority of tissues are taken by surgery or autopsy. In our case, the patient underwent Video-assisted thoracoscopic surgery. However, it will be beneficial to improve patients' prognosis if PAS can be diagnosed preoperatively. Some single centers showed that endovascular catheter biopsy was an effective and safe diagnostic tool for patients with PAS. ${ }^{11,12}$ Endovascular catheter biopsy may be repeated to obtain the real lesion site, since the tumor tissue of PAS is always covered with necrotic tissues or in situ thrombus. When suspected with PAS, an endovascular catheter biopsy should be performed to make an early diagnosis. Another study with a small number of cases suggested that directional atherectomy provides more sufficient material for histological analyses, and the rate of false negative results was $0 \%$. It seems that directional atherectomy could be a safer and more feasible technique to provide endovascular tumor sampling, including pulmonary artery sarcoma. ${ }^{13}$

In addition, there are a few reports of a pathological diagnosis obtained using endobronchial ultrasound-guided transbronchial needle aspiration (EBUS-TBNA). These studies have shown that EBUS-TBNA is a possible and less invasive method to evaluate endovascular lesions, including PAS. ${ }^{14,15}$ In the diagnosis of PAS, the limited sample is the challenge of EBUS-TBNA . Cytological material obtained by EBUS-TBNA with rapid on-site evaluation (ROSE) helped in the accurate early diagnosis of PAS. ${ }^{3,16}$ Supported by EBUS-TBNA with ROSE, the patient received preoperative chemotherapy, and surgical 
resection with reconstruction EBUS-TBNA can be used as a diagnostic option for PAS according to the tumor location. Overall, there is limited literature on the diagnosis of PAS by EBUS-TBNA.

Arising from the pluripotent mesenchymal stem cells of the intimal vessel layer, PAS has various complex pathological types. According to the 2015 World Health Organization classification of tumors, the most common pathological types of PAS patients include undifferentiated sarcoma, rhabdomyosarcoma, osteogenic sarcoma, angiosarcoma, fibrosarcoma, malignant mesenchymoma, myxosarcoma, chondrosarcoma osteosarcoma, malignant fibrous histiocytoma liposarcoma and unclassified leiomyosarcomas. ${ }^{17,18}$ The tumor of our patient was strongly positive for desmin and vimentin but negative for CD34, SMA, HMB45, MyoD1 and myogenin. This finding suggested that the histological type of our PAS patient is undifferentiated sarcoma, which is probable for a myogenic malignant tumor.

Since there are no clinical trials with PAS patients, therapeutic decisions are based on reported cases. Surgical resection is the main treatment for patients with PAS. It offers the best chance to prolong patient survival. However, the present patient was diagnosed with PAS with pulmonary metastasis. In addition, the family and the patient did not want surgery treatment because of the risk. Thus, whole exome sequencing was performed to identify the therapeutic target and genetic susceptibility genes. This sequencing contained targeted therapy-related genes, including KIT CNV, PIK3CA and TP53 mutations. The indices of the efficacy of immunological checkpoint inhibitors, including TMB MMR PD-L1, were all negative. Since the MTHFR rs1801133 polymorphism indicated that the patient might be sensitive to pemetrexed, the patient received a chemotherapy regimen of pemetrexed. However, clinical manifestations were aggravative, and the patient accepted to try anlotinib.

Anlotinib is a newly developed oral tyrosine kinase inhibitor that targets vascular endothelial growth factor receptor (VEGFR), fibroblast growth factor receptor (FGFR), platelet-derived growth factor receptor (PDGFR), and c-kit. As presented in the oral report at the American Society of Clinical Oncology (ASCO) annual meeting, anlotinib has excellent therapeutic efficacy in advanced soft tissue sarcoma. ${ }^{19}$ However, to date, there is no literature about anlotinib as a treatment for patients with PAS. After receiving anlotinib following chemotherapy, lung metastasis lesions were controlled, and pulmonary artery pressure was lower. However, due to a tumor burden and multiple metastases, the patient eventually died. PAS is a rare and difficult disease to diagnose, and it is challenging to conduct large-sample, randomized, double-blind, multicenter clinical trials of target therapy for PAS.

Given the low incidence of the disease, the molecular mechanism and origin of PAS is largely unknown. Genomic analysis showed that the amplification of $M D M 2, K I T$, and PDFGRA in PAS and patients could benefit from therapies targeting PDGFRA or $M D M 2 .^{20,21}$ Several investigators have reported that pulmonary artery sarcoma has MDM2 amplification and PDGFR $\alpha$ overexpression in PAS. ${ }^{16,22}$ The MDM2-p53 pathway is strongly associated with the tumorigenesis of pulmonary artery intimal sarcomas. ${ }^{23,24}$ Tovar $\mathrm{C}$ et al. offered preclinical evidence that the MDM2 small-molecule antagonist RG7112 inhibited human tumors through p53 signaling. ${ }^{25}$ Furthermore, ex vivo immunoassays on primary PAS cells from one patient showed the potency of dasatinib to inhibit PDGFRA and downstream MAPK and AKT signaling pathways. $^{26}$ In our case, $\mathrm{mdm} 2$ and P53 were also expressed in tumor cells of PAS by immunohistochemistry. Strikingly, whole exome sequencing of the tissue of this patient identified genetic alterations to the profile of PAS: KIT CNV and mutation of TP53, PIK3CA, IL7R, $A T R$. This case illustrates the phenotypic variation of PAS and may have implications for treatment. Further studies are required to explore biological markers or therapeutic targets in pulmonary artery sarcoma.

\section{Conclusion}

PAS is a class of rare and aggressive malignant tumors with no specific symptoms and that are easily misdiagnosed as PTE. For this reason, pathogenesis, molecular mechanism, diagnosis, treatment, and follow-up are often major challenges for patients with PAS. PET/CT with increased ${ }^{18}$ FDG uptake value can contribute to an accurate diagnosis for determining PA malignancy. Sequencing of PAS may have implications for molecular mechanism and treatment. Thus, we hope that this review and report of a patient with PAS will emphasize the recognition of this disease.

\section{Ethical approval}

This study was approved by the Ethics Committee of Zhongda Hospital, School of Medicine, Southeast University (Nanjing, China). The patient provided written informed consent for the case details and images to be published. 


\section{Acknowledgments}

We sincerely thank Lihua Zhang and Guoqing Wang, Department of Pathology, Zhongda Hospital, School of Medicine, Southeast University, Nanjing, for providing help in immunohistochemistry. This work was supported by grants from the National Natural Science Foundation of China (grant number: 81702279).

\section{Disclosure}

The authors report no conflicts of interest in this work.

\section{References}

1. David S, Hoeper MM, Vogel-Claussen J, Cebotari S. Pulmonary arterial sarcoma presenting as acute pulmonary embolism. Am J Respir Crit Care Med. 2017;196(4):523. doi:10.1164/rccm.201703-0596IM

2. Tueller C, Fischer Biner R, Minder S, et al. FDG-PET in diagnostic work-up of pulmonary artery sarcomas. Eur Respir J. 2010;35 (2):444-446. doi:10.1183/09031936.00114708

3. Caraway NP, Salina D, Deavers MT, Morice R, Landon G. Pulmonary artery intimal sarcoma diagnosed using endobronchial ultrasound-guided transbronchial needle aspiration. CytoJournal. 2015;12:3. doi:10.4103/1742-6413.151667

4. Wong HH, Gounaris I, McCormack A, et al. Presentation and management of pulmonary artery sarcoma. Clin Sarcoma Res. 2015;5 (1):3. doi:10.1186/s13569-014-0019-2

5. Li J, Zhao Q, He L, Zhuang X, Li F. Primary pulmonary artery sarcoma on dual-time point FDG PET/CT imaging. Clin Nucl Med. 2016;41(8):656-658. doi:10.1097/RLU.0000000000001248

6. Kessler A, Son H. Pulmonary artery angiosarcoma on 18F-FDG PET/ CT masquerading as pulmonary embolism. Clin Nucl Med. 2015;40 (1):82-84. doi:10.1097/RLU.0000000000000614

7. Hmelik S, Dobrenic M, Huic D. F-18 FDG PET/CT in pulmonary artery sarcoma: clinical vignette. Nucl Med Rev Cent East Eur. 2018;21(1):48-49. doi:10.5603/NMR.a2018.0011

8. Guo Y, Wang T, Yang M. Pulmonary artery sarcoma detected on $18 \mathrm{~F}-$ FDG PET/CT with unusual findings. Clin Nucl Med. 2015;40(11): e530-e531. doi:10.1097/RLU.0000000000000945

9. Lee DH, Jung TE, Lee JH, Shin DG, Park WJ, Choi JH. Pulmonary artery intimal sarcoma: poor $18 \mathrm{~F}$-fluorodeoxyglucose uptake in positron emission computed tomography. $J$ Cardiothorac Surg. 2013;8:40. doi:10.1186/1749-8090-8-40

10. Xi XY, Gao W, Gong JN, et al. Value of (18)F-FDG PET/CT in differentiating malignancy of pulmonary artery from pulmonary thromboembolism: a cohort study and literature review. Int $J$ Cardiovasc Imaging. 2019. doi:10.1007/s10554-019-01553-5

11. Kwon BS, Park JH, Gwon DI, Lee JS. Feasibility of endovascular catheter biopsy as a diagnostic modality for patients with pulmonary artery sarcoma: case series of a single-center experience. Vasc Endovascular Surg. 2018;52(8):636-640. doi:10.1177/1538574418779470
12. Xie WM, Zhai ZG, Wang LF, Wan J, Yang YH, Wang C. Endovascular catheter-guided forceps biopsy for the diagnosis of suspected pulmonary artery sarcoma: a preliminary study of eight cases. Chin Med J. 2016;129(18):2246-2249. doi:10.4103/0366-6999.189910

13. Massmann A, Seidel R, Schneider GK, Buecker A, Fries P. Percutaneous endovascular tissue sampling of endoluminal tumors using directional atherectomy. Eur Radiol. 2019. doi:10.1007/s00330-019-06015-z

14. Al-Saffar F, Ibrahim S, Seeram V, Bajwa AA, Shujaat A. Use of endobronchial ultrasound to evaluate nonthrombotic endovascular lesions in pulmonary arteries: a systematic review. J Bronchology Interv Pulmonol. 2015;22(1):28-32. doi:10.1097/LBR.0000000000000133

15. Romero Frances L, Royo Prats JA. Pulmonary artery leiomyosarcoma diagnosed by magnetic resonance, PET-CT and EBUS-TBNA. Arch Bronconeumol. 2017;53(9):522-523. doi:10.1016/j.arbres.2017.01.019

16. Harbhajanka A, Dahoud W. Cytohistological correlation, immunohistochemistry and Murine Double Minute Clone 2 amplification of pulmonary artery intimal sarcoma: A case report with review of literature. Diagn Cytopathol. 2019;47(5):494-497. doi:10.1002/dc.24131

17. Blackmon SH, Rice DC, Correa AM, et al. Management of primary pulmonary artery sarcomas. Ann Thorac Surg. 2009;87(3):977-984. doi:10.1016/j.athoracsur.2008.08.018

18. Travis WD, Brambilla E, Burke AP, Marx A, Nicholson AG. Introduction to the 2015 World Health Organization classification of tumors of the lung, pleura, thymus, and heart. J Thorac Oncol. 2015;10(9):1240-1242. doi:10.1097/JTO.0000000000000663

19. Chi Y, Fang Z, Hong X, et al. Safety and efficacy of anlotinib, a multikinase angiogenesis inhibitor, in patients with refractory metastatic soft-tissue sarcoma. Clin Cancer Res. 2018;24(21):5233-5238. doi:10.1158/1078-0432.CCR-17-3766

20. Neuville A, Collin F, Bruneval P, et al. Intimal sarcoma is the most frequent primary cardiac sarcoma: clinicopathologic and molecular retrospective analysis of 100 primary cardiac sarcomas. Am J Surg Pathol. 2014;38(4):461-469. doi:10.1097/PAS.0000000000000184

21. Zhao J, Roth J, Bode-Lesniewska B, Pfaltz M, Heitz PU, Komminoth P. Combined comparative genomic hybridization and genomic microarray for detection of gene amplifications in pulmonary artery intimal sarcomas and adrenocortical tumors. Genes Chromosomes Cancer. 2002;34(1):48-57. doi:10.1002/(ISSN)1098-2264

22. Takemoto S, Soda H, Iwasaki K, et al. Pulmonary artery sarcoma overexpressing platelet-derived growth factor receptor alpha. Intern Med. 2017;56(7):823-826. doi:10.2169/internalmedicine.56.7731

23. Bode-Lesniewska B, Zhao J, Speel EJ, et al. Gains of 12q13-14 and overexpression of $\mathrm{mdm} 2$ are frequent findings in intimal sarcomas of the pulmonary artery. Virchows Arch. 2001;438(1):57-65.

24. Stojsic J, Popovic M, Adzic-Vukicevic T, et al. Rare histological subtype of pulmonary artery intimal sarcoma diagnosed by multidisciplinary approach. J Res Med Sci. 2018;23:70. doi:10.4103/jrms.JRMS_102_18

25. Tovar C, Graves B, Packman K, et al. MDM2 small-molecule antagonist RG7112 activates p53 signaling and regresses human tumors in preclinical cancer models. Cancer Res. 2013;73(8):2587-2597. doi:10.1158/0008-5472.CAN-12-2807

26. Dewaele B, Floris G, Finalet-Ferreiro J, et al. Coactivated plateletderived growth factor receptor \{alpha\} and epidermal growth factor receptor are potential therapeutic targets in intimal sarcoma. Cancer Res. 2010;70(18):7304-7314. doi:10.1158/0008-5472.CAN-10-1543 


\section{Publish your work in this journal}

OncoTargets and Therapy is an international, peer-reviewed, open access journal focusing on the pathological basis of all cancers, potential targets for therapy and treatment protocols employed to improve the management of cancer patients. The journal also focuses on the impact of management programs and new therapeutic agents and protocols on patient perspectives such as quality of life, adherence and satisfaction. The manuscript management system is completely online and includes a very quick and fair peer-review system, which is all easy to use. Visit http://www.dovepress.com/ testimonials.php to read real quotes from published authors.

Submit your manuscript here: https://www.dovepress.com/oncotargets-and-therapy-journal 